\title{
Building Intentional Models Using the ERi*c Method
}

\author{
Antonio de Padua Albuquerque Oliveira ${ }^{1}$, Julio Cesar Sampaio do Prado Leite ${ }^{2}$ \\ ${ }^{1}$ Universidade do Estado do Rio de Janeiro - UERJ \\ ${ }^{2}$ Departamento de Informática da Pontificia Universidade Católica do Rio de Janeiro - \\ PUC-Rio
}

www.ime.uerj.br/ padual - www.inf.puc-rio.br/ julio

\begin{abstract}
Requirements elicitation is the backbone of modeling. People around the world do not give very much attention to early requirements elicitation and more precisely to actors' goals elicitation before eliciting and modeling requirements. This fault will incur in several losses either during the development, or worse, later. Goals \{flexible (softgoals) and concrete\} are the foundation of GORE and consequently of $i^{*}$. The ERi*c Method goal elicitation maturity was consolidated by its use in several academic projects and can support not only to the entire requirements process, but also to reengineer, to change the model of a system, in order to include the use of new technology. This paper shows the strength of the ERi*c Method using as an example a published $i *$ SR model. It also focuses on how the ERi*c Method can split an SR model of an Information System into several comprehensible models, applying the concept of SDsituations (Strategic Dependency situations).
\end{abstract}

Keywords: goals, early requirements, elicitation, modeling, GORE, Goal Oriented Requirements Engineering, ERi*c, goal modeling.

\section{Introduction}

The Eri*c method was proposed by Oliveira [3] and has been evolving as it has been used by students and practitioners over the years. We have published parts of the ERi*c method [2] [4] [5] and in this paper we focus on the cornerstone of ERi*c: that is the elicitation strategy and the modularization strategy in their latest versions. We have used a previous published model as an example, as a way, to help communicating our ideas.

\section{Objectives of the research}

Since the publication of Oliveira's thesis, we have concentrated on improving ERi*c to support the construction of intentional models, as basis for our software transparency effort [6]. The method has been taught at two universities and it has been applied to several case studies as Zipcar (a car-sharing system), embedded systems, and also in a financial system for a very large telecom company. 
In the next section we explain in a concise way the latest version of the process used in the ERi*c Method to identify goals. The aim is to show how to build comprehensible $i^{*}$ models departing of an SR model from another author.

\subsection{Actors' goals elicitation}

Using only one source of information is not the best practice, (see Figure 1) but, in our case, given the constraints and our purpose, the SR model published in [1] is sufficient ${ }^{1}$. Our purpose is to exemplify how ERi*c can re-engineer one previous SR diagram in order to mitigate the complexity by controlling the scalability.

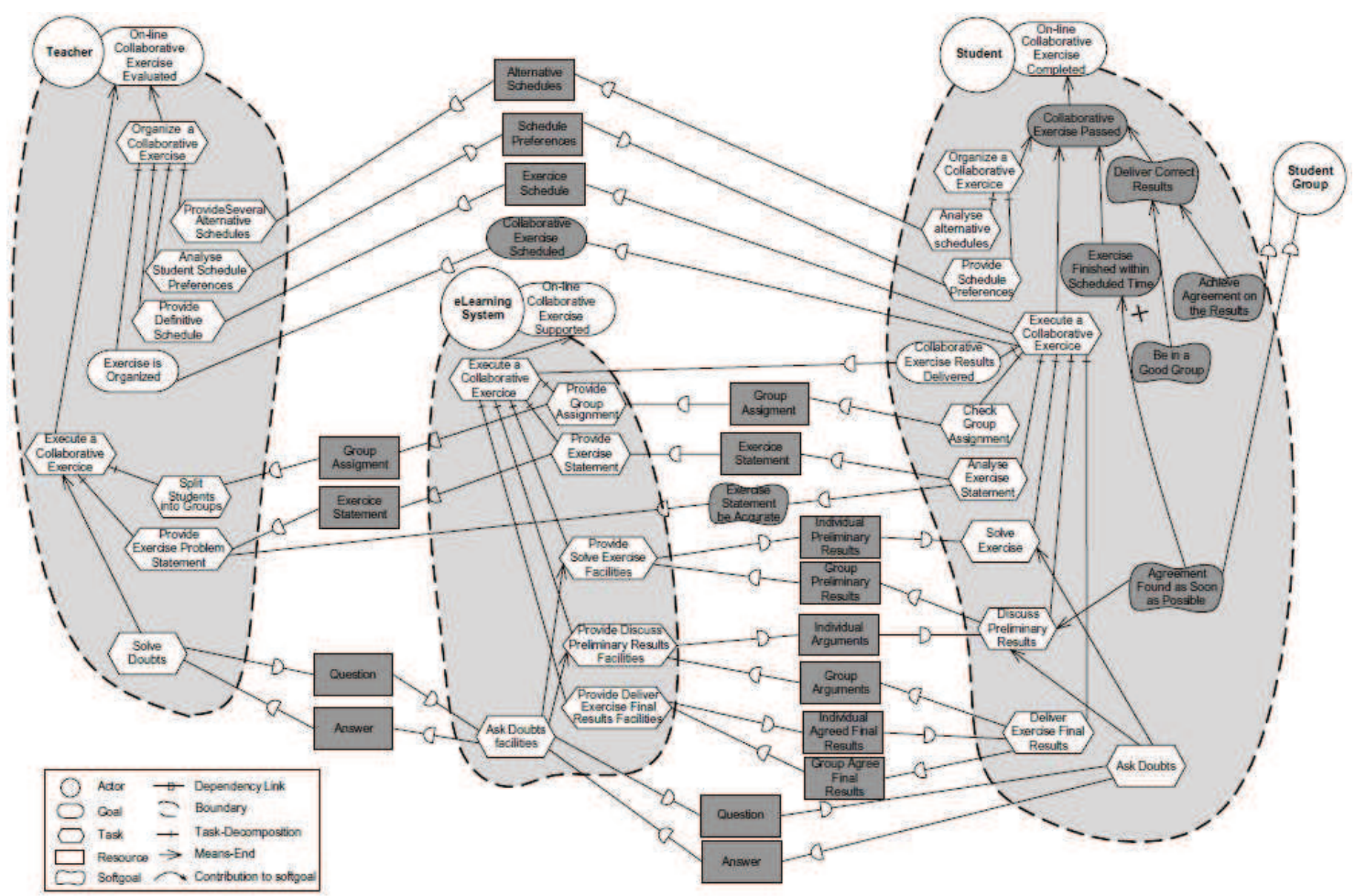

Figure 1 - The SR model that was used as source of information [1]

We will show that that our choice was adequate to demonstrate how simple the ERi*c process is. Steps: (i) identify the list of relevant symbols which are words or peculiar sentences mostly used; (ii) classify symbols as: subject, object, verb, and state; (iii) describe symbols through notions and behavioral responses definitions [2]. In Table 1, applying the 3 steps above; we selected actors' (teacher and student) tasks as behavioral responses and objects in order to give us more behavioral responses.

\footnotetext{
${ }^{1}$ It will be clear that common sense knowledge is used as to fill the gaps of information provided by the chosen information source.
} 
Table 1 -Actors' tasks and resources from the SR model [1]

\begin{tabular}{|l|l|l|}
\hline TEACHER (SUBJECT) & STUDENT (SUBJECT) & RESOURCES $\rightarrow$ (OBJECT) \\
\hline Organize a collaborative exercise & Organize collaborative exercise & alternative schedules \\
\hline Provide alternative schedules & Analyse alternative schedules & schedule preferences \\
\hline Analyse students schedule preferences & Provide schedule preferences & exercise schedule \\
\hline Provide a definitive schedule & Execute collaborative exercise & group assignment \\
\hline Split students into groups & Check group assignment & exercise statement \\
\hline Execute a collaborative exercise & Analyse exercise statement & question \\
\hline Provide exercise problem statement & Solve exercise & answer \\
\hline Solve doubts & Discuss preliminary results & Individual preliminary results \\
\hline Provide group assignment & Deliver exercise final results & group preliminary results \\
\hline Provide exercise statement & Ask doubts & individual arguments \\
\hline Provide solve exercise facilities & & group arguments \\
\hline Provide discuss preliminary results facilities & & individual agreed final results \\
\hline Provide exercise final results facilities & & group agree final results \\
\hline Ask doubts facilities & & \\
\hline
\end{tabular}

"Behavioral Responses" (BRs) declare actions. Two kinds of actions can be observed: concrete and flexible. A concrete action brings one concrete result, and a flexible action brings a quality attribute [2], [3]. The key point of the template in Table 2 is to identify the motivation, the intentionality (why?) under the BR. "Why does teacher define schedule?" - is an example. The verbs to organize, to offer, and to analyse are flexible actions examples as well as "solves doubts" and discusses.

Table 2 - Teacher's Goals from behavioral responses with concrete actions

\begin{tabular}{|c|c|c|c|c|c|}
\hline \multirow{2}{*}{$\begin{array}{l}\text { SYMBOL (type: subject) } \\
\text {-- behavioral response }\end{array}$} & \multicolumn{3}{|c|}{ <concrete goal> } & \multirow[b]{2}{*}{ by } & \multirow{2}{*}{$\begin{array}{c}\text { actor } \\
\text { <subject LEL: }\end{array}$} \\
\hline & <subject / object LEL> & BE & <verb> & & \\
\hline \multicolumn{6}{|l|}{ TEACHER } \\
\hline$\rightarrow$ Organizes a collaborative exercise. & flexible action & & & & \\
\hline$\rightarrow$ Offers several alternative schedules & flexible action & & & & \\
\hline$\rightarrow$ Analises students schedule preferences. & flexible action & & & & \\
\hline \multicolumn{6}{|l|}{$\rightarrow$ Defines the definitive schedule. } \\
\hline BECAUSE teacher wants & schedule & $\mathrm{BE}$ & created & by & student \\
\hline \multicolumn{6}{|l|}{$\rightarrow$ Executes a collaborative exercise. } \\
\hline BECAUSE teacher wants & exercise & $\mathrm{BE}$ & learned & by & student \\
\hline BECAUSE student wants & exercise & $\mathrm{BE}$ & evaluated & by & teacher \\
\hline \multicolumn{6}{|l|}{$\rightarrow$ Splits students into groups. } \\
\hline BËCAUSE teacher wants & group & $\mathrm{BE}$ & organized & by & student \\
\hline BECAUSE student wants & group & $\mathrm{BE}$ & created & by & teacher \\
\hline$\rightarrow$ Solves student doubts. & flexible action & & & & \\
\hline \multicolumn{6}{|l|}{$\rightarrow$ Provides group assignment. } \\
\hline BECAUSE teacher wants & exercise & $\mathrm{BE}$ & proposed & & \\
\hline \multicolumn{6}{|l|}{$\rightarrow$ Provide exercise statement. } \\
\hline BECAUSE teacher wants & exercise & $\mathrm{BE}$ & understood & by & group \\
\hline BECAUSE group wants & exercise & $\mathrm{BE}$ & evaluated & by & teacher \\
\hline \multicolumn{6}{|l|}{$\rightarrow$ Provide solve exercise facilities. } \\
\hline BECAUSE teacher wants & exercise & $\mathrm{BE}$ & understood & by & student \\
\hline BECAUSE student wants & exercise & $\mathrm{BE}$ & done & by & group \\
\hline BECAUSE student wants & exercise & $\mathrm{BE}$ & evaluated & by & teacher \\
\hline$\rightarrow$ Discusses preliminary results facilities. & flexible action & & & & \\
\hline \multicolumn{6}{|l|}{$\rightarrow$ Delivers exercise final results facilities. } \\
\hline BECAUSE teacher wants & results & $\mathrm{BE}$ & published & & \\
\hline \multicolumn{6}{|l|}{$\rightarrow$ Asks doubts facilities. } \\
\hline BECAUSE teacher wants & exercise & $\mathrm{BE}$ & understood & by & student \\
\hline BECAUSE student wants & exercise & $\mathrm{BE}$ & done & & \\
\hline
\end{tabular}


Following the process we show (Table 3) concrete goals elicitation. Flexible goals, which are elicited form flexible actions, are manipulated by the template showed by Table 5, after the elicitation of concrete goals from objects (Table 4).

\section{Table 3 - Student's Goals from behavioral responses with concrete actions}

\begin{tabular}{|c|c|c|c|c|c|}
\hline \multicolumn{6}{|l|}{ STUDENT } \\
\hline$\rightarrow$ Participates in a collaborative exercise. & flexible action & & & & \\
\hline$\rightarrow$ Analyses alternative schedules. & flexible action & & & & \\
\hline \multicolumn{6}{|l|}{$\rightarrow$ Chooses schedule preferences. } \\
\hline BECAUSE student wants & preferences & $\mathrm{BE}$ & considered & by & teacher \\
\hline BECAUSE teacher wants & schedule & $\mathrm{BE}$ & defined & & \\
\hline \multicolumn{6}{|l|}{$\rightarrow$ Executes collaborative exercise. } \\
\hline BECAUSE student wants & exercise & $\mathrm{BE}$ & evaluated & by & teacher \\
\hline BECAUSE teacher wants & exercise & $\mathrm{BE}$ & evaluated & & \\
\hline$\rightarrow$ Checks group assignment. & flexible action & & & & \\
\hline$\rightarrow$ Analyses exercise statement. & flexible action & & & & \\
\hline \multicolumn{6}{|l|}{$\rightarrow$ Solves exercise. } \\
\hline BECAUSE student wants & exercise & $\mathrm{BE}$ & evaluated & by & teacher \\
\hline BECAUSE teacher wants & results & $\mathrm{BE}$ & published & & \\
\hline$\rightarrow$ Discusses preliminary results. & flexible action & & & & \\
\hline \multicolumn{6}{|l|}{$\rightarrow$ Delivers exercise final results. } \\
\hline BECAUSE student wants & exercise & $\mathrm{BE}$ & evaluated & by & teacher \\
\hline BECAUSE teacher wants & results & $\mathrm{BE}$ & published & & \\
\hline \multicolumn{6}{|l|}{$\rightarrow$ Asks exercise doubts. } \\
\hline BECAUSE student wants & exercise & $\mathrm{BE}$ & understood & & \\
\hline
\end{tabular}

Table 4 -Concrete goals from objects behavioral responses

\begin{tabular}{|c|c|c|c|c|c|}
\hline SYMBOL (type: object) & <concre & goal> & & & actor \\
\hline$\rightarrow$ behavioral response & 〈subject/object LEL $\rangle$ & $\mathrm{BE}$ & $\langle$ verb $\rangle$ & by & 〈subject LEL> \\
\hline ALTERNATIVE SCHEDULES & & & & & \\
\hline$\rightarrow$ They are communicated by teacher & preferences & $\mathrm{BE}$ & considered & by & student \\
\hline SCHEDULE PREFERENCES & & & & & \\
\hline$\rightarrow$ Student choose the best schedule. & flexible action & & & & student \\
\hline EXERCISE SCHEDULE & & & & & \\
\hline$\rightarrow$ Is elaborated by teacher. & schedule & $\mathrm{BE}$ & obeyed & by & student \\
\hline$\rightarrow$ Must be obeyed by students. & exercise & $\mathrm{BE}$ & evaluated & by & teacher \\
\hline GROUP ASSIGNIMENT & & & & & \\
\hline$\rightarrow$ Student group receive the assignment. & exercise & $\mathrm{BE}$ & done & by & student \\
\hline$\rightarrow$ Is defined by teacher. & group & $\mathrm{BE}$ & created & by & teacher \\
\hline EXERCISE STATEMENT & & & & & \\
\hline$\rightarrow$ Appears in the assignment. & exercise & $\mathrm{BE}$ & done & by & student \\
\hline$\rightarrow$ Is made by teacher. & exercise & $\mathrm{BE}$ & done & by & student \\
\hline QUESTION & & & & & \\
\hline$\rightarrow$ Student asks doubt in the exercise. & flexible action & & & & \\
\hline ANSWER & & & & & \\
\hline$\rightarrow$ Teacher answers doubt in the exercise. & flexible action & & & & student \\
\hline INDIVIDUAL PRELIMINARY RESULTS & & & & & \\
\hline$\rightarrow$ Student solves exercise. & exercise & $\mathrm{BE}$ & done & by & student \\
\hline$\rightarrow$ Should be distributed for the group. & results & $\mathrm{BE}$ & published & & teacher \\
\hline GROUP PRELIMINARY RESULTS & & & & & \\
\hline$\rightarrow$ Should receive the agreement of the group. & flexible action & & & & \\
\hline INDIVIDUAL ARGUMENTS & & & & & \\
\hline$\rightarrow$ Student discusses preliminary results. & flexible action & & & & \\
\hline$\rightarrow$ Can be distributed for the group. & Solution & $\mathrm{BE}$ & discussed & by & student \\
\hline GROUP ARGUMENTS & & & & & \\
\hline$\rightarrow$ Student discusses preliminary results. & flexible action & & & & \\
\hline$\rightarrow$ Can be distributed for the group. & solution & $\mathrm{BE}$ & discussed & by & group \\
\hline INDIVIDUAL AGREED FINAL RESULTS & & & & & \\
\hline$\rightarrow$ Student agree with results. & flexible action & & & & \\
\hline$\rightarrow$ Final results should receive an agreement. & flexible action & & & & \\
\hline GROUP AGREE FINAL RESULTS & & & & & \\
\hline$\rightarrow$ Final results is sent to teacher. & exercise & $\mathrm{BE}$ & evaluated & by & teacher \\
\hline$\rightarrow$ Final results should obey schedule. & flexible action & & & & \\
\hline
\end{tabular}

\section{Flexible goals}

After identifying concrete goals we should identify flexible goals [3]. When one concrete action is found $\rightarrow$ the action will define a concrete goal. When one flexible action is found $\rightarrow$ the action will define a flexible goal. Eric Yu's thesis defined: "A goal is a condition or state of affairs in the world that an actor would like to achieve" 
and our basic idea is: "ACTIONS CHANGE STATES AND STATES ARE GOALS" [3].

The aim of the template (Table 5) is to identify the motivation under the flexible action, "Why does the teacher organize a collaborative exercise?" - is an example.

The idea is: flexible actions mean that quality attributes are the reason of each action.

Table 5 indicates the best concrete goal, which is associated to the flexible goal, and also the actor who has the goal responsibility.

Table 5 - Flexible goals elicited with concrete goals associated

\begin{tabular}{|c|c|c|c|c|}
\hline \multirow{2}{*}{$\begin{array}{l}\text { SYMBOL } \\
\rightarrow \text { behavioral response } \\
\text { flexible action } \quad \text { WHY ? }\end{array}$} & \multicolumn{2}{|c|}{ FLEXIBLE GOAL } & \multirow[b]{2}{*}{$\langle$ concrete goal associated $>$} & \multirow[b]{2}{*}{ <actor> } \\
\hline & <type > & $\langle[$ topic $]\rangle$ & & \\
\hline \multicolumn{5}{|l|}{ Teacher } \\
\hline Organizes a collaborative exercise. BECAUSE & quality & [solution] & exercise BE shared & group \\
\hline Offers several alternative schedules. BECAUSE & fare & [schedule] & schedule BE created & student \\
\hline Analises students schedule preferences. BECAUSE & proper & [delivery] & preferences BE considered & teacher \\
\hline Solves student doubts. BECAUSE & accurate & [solution] & exercise BE learned & teacher \\
\hline Discusses preliminary results facilities. BECAUSE & proper & [exercise] & exercise BE done & teacher \\
\hline Teacher answers doubt in the exercise. BECAUSE & accurate & [solution] & exercise BE learned & teacher \\
\hline \multicolumn{5}{|l|}{ Student } \\
\hline Participates in a collaborative exercise. BECAUSE & uality & [solution] & exercise BE done & student \\
\hline Analyses alternative schedules. BECAUSE & fare & [schedule] & exercise BE done & student \\
\hline Checks group assignment. BECAUSE & reliable & [group] & group BE created & teacher \\
\hline Analyses exercise statement. BECAUSE & reliable & [exercise] & xercise BE understood & student \\
\hline Discusses preliminary results. BECAUSE & quality & [solution] & exercise BE done & student \\
\hline Student choose the best schedule. BECAUSE & good & [schedule] & schedule BE obeyed & student \\
\hline Student asks doubt in the exercise. BECAUSE & quality & [solution] & exercise BE done & student \\
\hline Should receive the agreement of the group. BECAUSE & quality & [solution] & exercise BE done & student \\
\hline Student discusses preliminary results. BECAUSE & quality & [solution] & exercise BE done & student \\
\hline \multicolumn{5}{|l|}{ Group } \\
\hline Student discusses preliminary results.. BEC & uality & [solution] & solution BE discussed & group \\
\hline Student agree with results. BECAUSE & quality & [solution] & exercise BE done & group \\
\hline Final results should obey schedule. BECAUSE & reliable & [schedule] & exercise BE evaluated & group \\
\hline
\end{tabular}

\subsection{Actors' goals refining}

At this point, the ERi*c Method proposes two activities: merge goals (concrete and flexible) by actor and set them in a chronological order. Chronological order means: long term goals first (the most abstract before and the less abstract after). During this activity we should delete duplicated goals; they do not help the process.

\subsection{SDsituation identification}

Definition: "An SDsituation is a dependency construct with one situational intentionality (one common goal) which is temporarily shared by some actors "[3].

Figure 2 illustrates the organizational components cycle. SDsituations should be identified by goals in the business part. Each frontier between two SDsituations can be discovered because a time interruption occurs during two SDsituations in the cycle. 


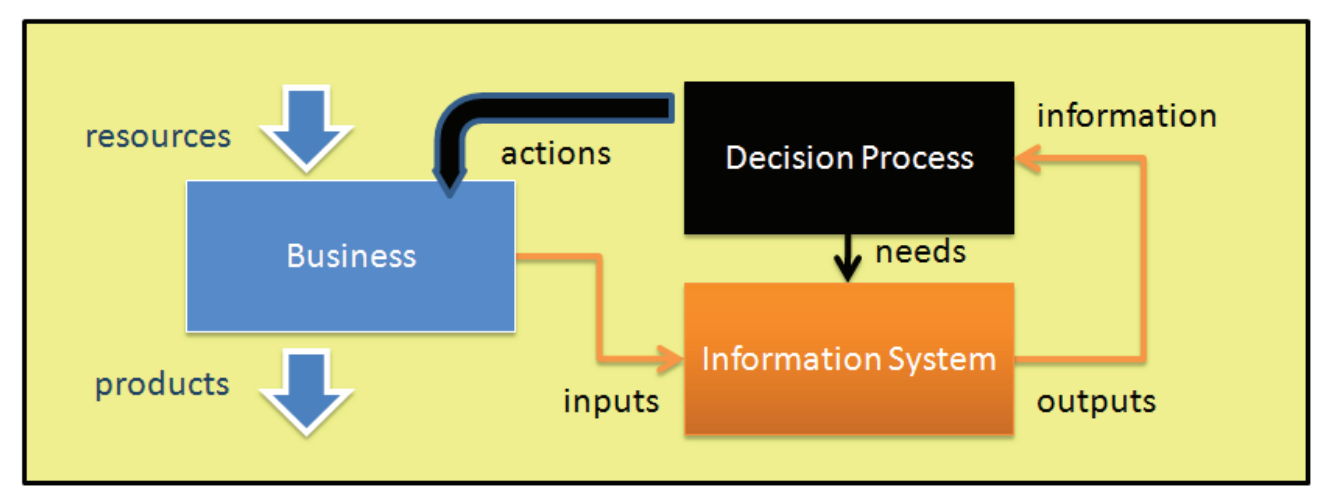

Figure 2 - Organizational Components Relationship Schema

The inputs of the IS are time stamped and the outputs are produced after some time slice. The outputs of the IS are the information about the "world" that the decision process needs to act on the business in order to improve business results. As such, five SDsituations were distinguished (Table 6): (1) Groups Organization, (2) Schedule Definition, (3) Exercise Proposition, (4) Exercise Solution, and (5) Exercise Evaluation.

Table 6 - Goals organized by actors in a chronological order

\begin{tabular}{|c|c|c|c|c|c|c|}
\hline DEPENDER & \multicolumn{5}{|c|}{ (SDsituation) } & \multirow[t]{2}{*}{ DEPENDEE } \\
\hline \multicolumn{6}{|l|}{ teacher } & \\
\hline \multirow[t]{2}{*}{ accurate [solution] } & (5) & exercise & $\mathrm{BE}$ & learned & by & student \\
\hline & (5) & results & $\mathrm{BE}$ & published & & \\
\hline \multirow[t]{6}{*}{ easy [delivery] } & (5) & exercise & $\mathrm{BE}$ & evaluated & & \\
\hline & (4) & exercise & $\mathrm{BE}$ & done & by & group \\
\hline & (4) & schedule & $\mathrm{BE}$ & obeyed & by & group \\
\hline & (3) & exercise & $\mathrm{BE}$ & understood & by & group \\
\hline & (3) & exercise & $\mathrm{BE}$ & understood & by & student \\
\hline & (3) & exercise & $\mathrm{BE}$ & proposed & & \\
\hline fare [schedule] & (2) & schedule & $\mathrm{BE}$ & defined & & \\
\hline proper [delivery] & (2) & preferences & $\mathrm{BE}$ & considered & & \\
\hline \multirow{2}{*}{ reliable [group] } & (1) & group & $\mathrm{BE}$ & created & & \\
\hline & (1) & group & $\mathrm{BE}$ & organized & by & student \\
\hline \multicolumn{7}{|l|}{ student } \\
\hline & (5) & results & $\mathrm{BE}$ & published & by & teacher \\
\hline & (5) & solution & $\mathrm{BE}$ & discussed & & \\
\hline & (5) & exercise & $\mathrm{BE}$ & evaluated & by & teacher \\
\hline \multirow[t]{2}{*}{ quality [solution] } & (4) & exercise & $\mathrm{BE}$ & done & & \\
\hline & (4) & schedule & $\mathrm{BE}$ & obeyed & & \\
\hline \multirow[t]{4}{*}{ reliable [exercise] } & (3) & exercise & $\mathrm{BE}$ & understood & & \\
\hline & (2) & schedule & $\mathrm{BE}$ & defined & by & teacher \\
\hline & (2) & preferences & $\mathrm{BE}$ & considered & & \\
\hline & (1) & group & $\mathrm{BE}$ & organized & & \\
\hline \multicolumn{7}{|l|}{ group } \\
\hline & (5) & exercise & $\mathrm{BE}$ & evaluated & by & teacher \\
\hline \multirow[t]{2}{*}{ quality [solution] } & (5) & solution & $\mathrm{BE}$ & discussed & & \\
\hline & (4) & exercise & $\mathrm{BE}$ & done & & student \\
\hline \multirow[t]{2}{*}{ quality [solution] } & (4) & exercise & $\mathrm{BE}$ & shared & & \\
\hline & (4) & schedule & $\mathrm{BE}$ & obeyed & & \\
\hline
\end{tabular}




\subsection{Modeling Actors' Goals}

This activity prepares one IP (Intentionality Panel) Diagram, one SD Model, and one SR Model for each SDsituation; but due to space limitation only one SDsituation is showed. Figure 3 shows the IP Diagram and Figure 4 shows the SR Model both from the same SDsituation (4) - Exercise Solution.

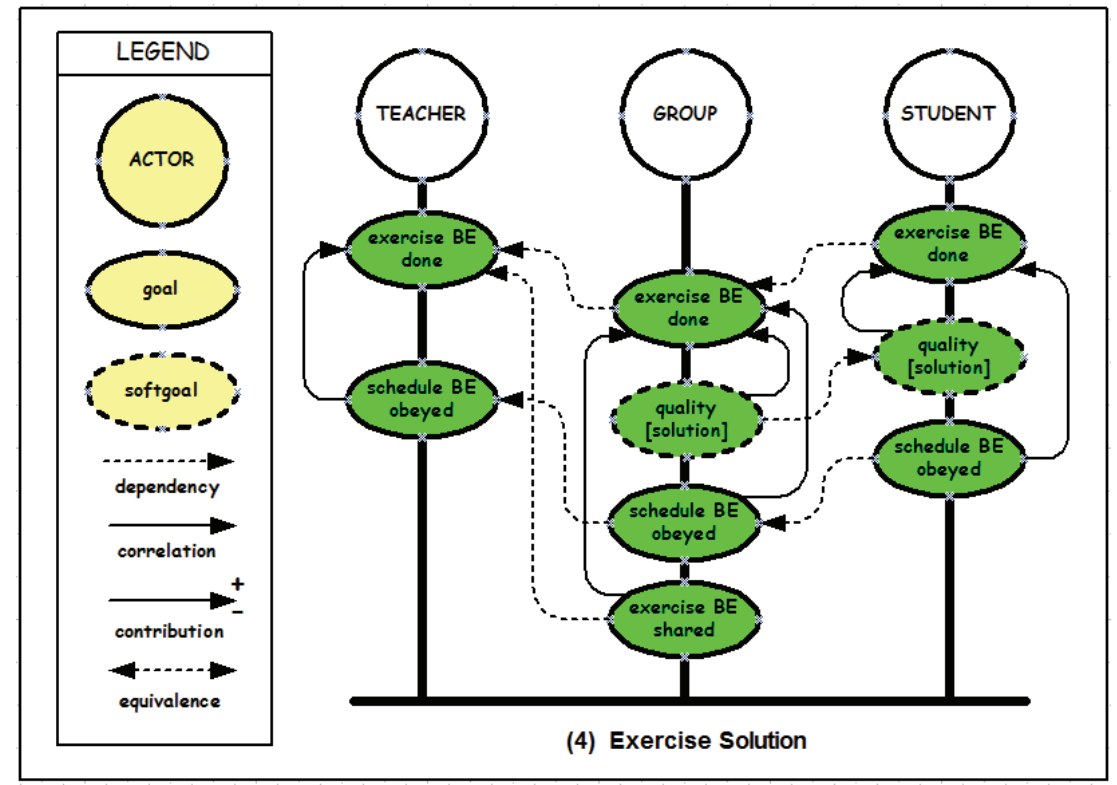

Figure 3 - SDsituation: (4) Exercise Solution - IP Diagram

One benefit of appling SDsituation concept is to deal with different situations in separated diagrams.

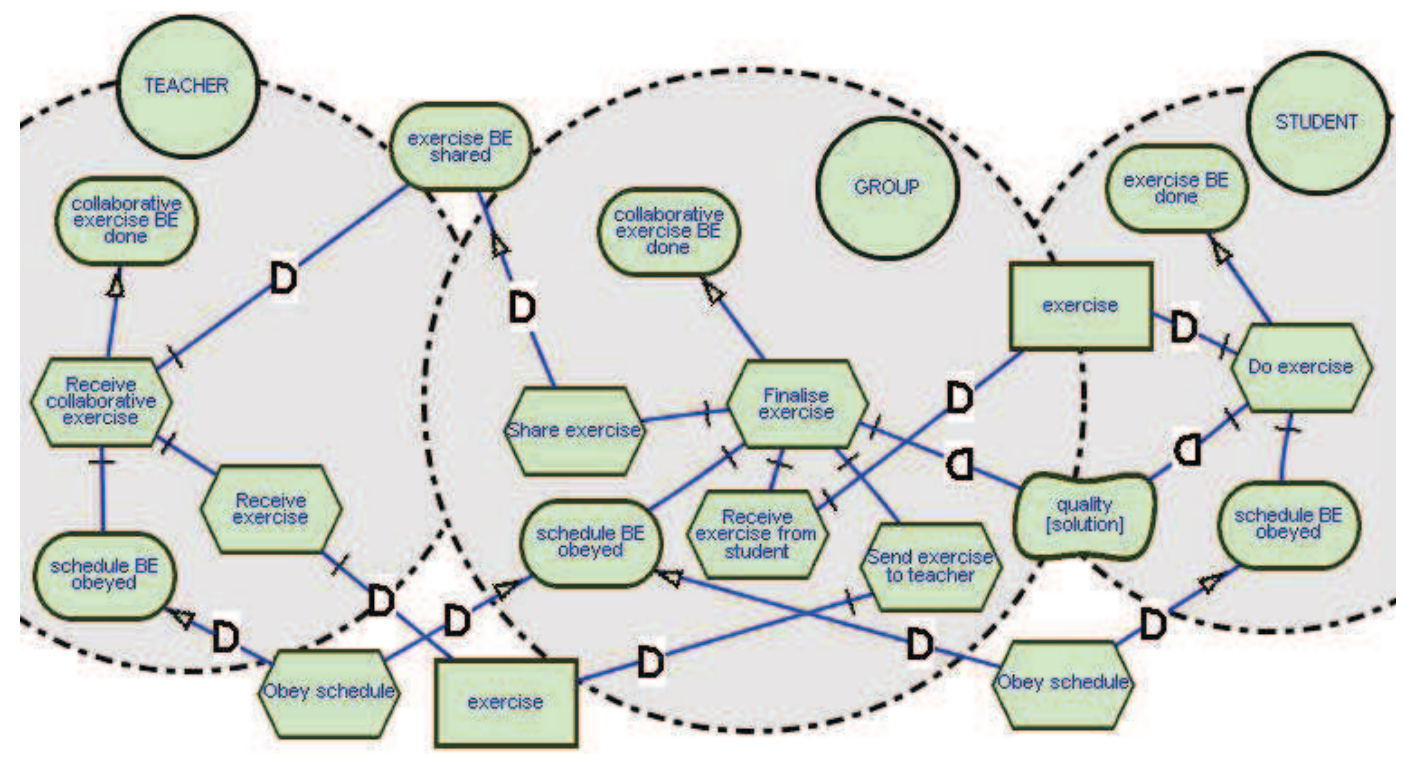

Figure 4 - SDsituation: (4) Exercise Solution -SR model 


\section{Scientific contributions}

The main goal of the ongoing research is to improve the method and to find ways of empowering requirements engineers to use intentional requirements engineering. In this paper we have focused on:

a.) Elicitation, which has been simplified consequently removing one step and one template, see Table 5; bringing more simplicity into the method.

b.) The SDsituations concept received more attention; we described more precisely the idea and therefore better results for student jobs.

c.) Instructions, heuristics, and examples were included in the whole method.

\section{Conclusion}

Intentional modeling can be the basis for software to be transparent. Expressing the desires of the social actors involved in a given context is a solid start point as to produce software artifacts that will not only perform what the client wants, but which also provides a solid ground for informing stakeholders about the software. We believe that the example discussed above does provide more detail about ERi*c, making it possible not only the publicity of the method, but also providing an opportunity for discussion over its limitations or appropriateness. It is mister to say that we are still trying to provide an environment that would automate some of its tasks.

\section{References}

[1] Grau, G.; Franch, X.; Maiden, N.; PRiM: An i*-based process reengineering method for information systems specification; ScienceDirect - Information and Software Technology 50 (2008) 76-100.

[2] Oliveira, Padua A.; Leite, J.; Cysneiros, L.; Cappelli, C.; "Eliciting Multi-Agents Systems Intentionality: From Language Extended Lexicon to i* Models", Proceedings of the 26th International Conference of the Chilean Computer Science Society. IEEE Computer Society, 2007. v16. p 40-49.

[3] Oliveira, Padua A.; (2008) Intentional Requirements Engineering: A Method for Requirements Elicitation, Modeling, and Analysis. 261p. Doctoral Thesis; Computer Science Department, PUC-Rio.

[4] Oliveira, Padua A.; Leite, J.; Cysneiros, L. (2010) Using i* Meta Modeling for Verifying i* Models. In: iStar 2010 4th International i* Workshop, Hammamet, Tunisia. CEUR - Workshop Proceedings. Aachen : rwth-aachen, 2010. v. 586. p. 7680 .

[5] Oliveira, Padua A.; Leite, J.; Cysneiros, L., Lucena, C.; i* Diagnoses: A Quality Process for Building i* Models pp. 9-12, CAiSE'08 Forum Proceedings of the Forum at the CAiSE'08 conference, p. 9-12

[6] Julio Cesar Sampaio do Prado Leite, Claudia Cappelli: Software Transparency. Business \& Information Systems Engineering 2(3): 127-139 (2010) 